\title{
Itinéraires
}

Itinéraires Littérature, textes, cultures

2012-1| 2012

Genres et avant-gardes

\section{Comment conjuguer un nouveau gender et de nouveaux genres}

Silvia Contarini

\section{(2) OpenEdition}

Journals

\section{Édition électronique}

URL : http://journals.openedition.org/itineraires/1238

DOI : $10.4000 /$ itineraires. 1238

ISSN : 2427-920X

Éditeur

Pléiade

\section{Édition imprimée}

Date de publication : 1 septembre 2012

Pagination : $35-48$

ISBN : 978-2-296-55776-5

ISSN : $2100-1340$

\section{Référence électronique}

Silvia Contarini, "Comment conjuguer un nouveau gender et de nouveaux genres », Itinéraires [En ligne], 2012-1 | 2012, mis en ligne le 01 septembre 2012, consulté le 01 mai 2019. URL : http:// journals.openedition.org/itineraires/1238; DOI : 10.4000/itineraires.1238

\section{(ब) $(\Theta \Theta$}

Itinéraires est mis à disposition selon les termes de la licence Creative Commons Attribution - Pas d'Utilisation Commerciale - Pas de Modification 4.0 International. 


\title{
Comment conjuguer un nouveau gender et de nouveaux genres
}

\begin{abstract}
In order to investigate the articulation between artistic pratice and gendered theorization of such pratice in the context of the futurist avant-garde, the present article analyzes the works of two futurist women, Rosa Rosà and Enif Robert. The hypothesis it develops is that gender issues are present in their work, and that both authors appropriate futurist principles in order to elaborate them in terms of gender, but that their artistic productions reveal even more so than their discourses the limits of their thinking on woman.
\end{abstract}

Keywords : Futurism, avant-garde, women writers, women, gender Mots clés : futurisme, avant-garde, écrivaines, femmes, genre sexué

À l'occasion de la célébration du centenaire de la naissance du futurisme, beaucoup de colloques et de publications ont été consacrés dans le monde à ce mouvement d'avant-garde. Très peu ont porté sur les femmes futuristes ${ }^{1}$; sans doute considère-t-on que depuis les travaux de Claudia Salaris sur les écrivaines, de Lea Vergine sur les femmes peintres ${ }^{2}$, et de

1. Outre la conférence sur les femmes futuristes que Simona Cigliana et moi-même avons donnée à l'Institut culturel du Caire le 31 mai 2009, je signale l'exposition « Futuriste. Women in Art and Literature », Casa Zerilli-Merimò, New York, 9 novembre-15 décembre 2009. Dans le texte de présentation de cette exposition, Futurist Women in America. An Exhibition and Research Opportunity, Giancarlo Carpi propose une lecture de la production féminine futuriste selon la catégorie esthétique de la cuteness, c'est-à-dire de la déformation du sujet iconographique (cf. http://www.futur-ism.it/esposizioni/ESP2009/ ESP20091109_NY.htm).

2. Claudia Salaris a réédité d'abord un roman de Rosa Rosà (Rosa Rosà, Una donna con tre anime: romanzo futurista, Milan, Edizioni delle donne, 1981), puis une anthologie d'œuvres d'une quarantaine d'écrivaines futuristes (Le Futuriste, donne e letteratura d'avanguardia in Italia (1909-1944), Milan, Edizioni delle donne, 1982). Quant à Lea Vergine, elle a organisé en 1980 l'exposition "L'altra metà dell'avanguardia », qui ne concerne pas les 
beaucoup d'autres qui, à partir des années 1980, ont réévalué la présence féminine au sein du futurisme, l' " autre moitié » est désormais intégrée à plein titre et que la question de l'articulation entre futurisme et gender ne mérite plus d'études spécifiques.

Il reste pourtant bien des aspects à creuser. Pour ma part, j'ai profité des sollicitations du centenaire pour développer quelques points abordés dans mon ouvrage La Femme futuriste ${ }^{3}$ et pour prolonger certaines conclusions auxquelles j'étais parvenue, notamment trois : l'existence d'un clivage assez net entre hommes et femmes futuristes quant à leur conception des relations de genre; l'existence d'apories dans les propos des uns et des autres; l'échec de l'élaboration d'un nouveau modèle de femme du futur en même temps que l'échec de l'ambition utopique du futurisme de reconstruire l'univers ${ }^{4}$.

Pour avancer dans ma réflexion, je me propose de revenir ici sur les liens entre pratique artistique et théorisation " sexuée » de cette pratique, entre œuvre et intentionnalité de l'œuvre (comme le soulignait Roland Barthes, la qualité évidente des textes d'avant-garde « est d'ordre intentionnel : ils s'empressent de servir la théorie $\left.{ }^{5} \gg\right)$. Mon hypothèse est que certaines femmes reconfigurent les préceptes futuristes en les adaptant à une spécificité de gender assumée, que leur interrogation sur le gender est présente aussi dans les formes et les thèmes, mais que le résultat artistique - aussi novateur et intéressant soit-il - dévoile, plus encore que leur discours, les limites de leur conception de la femme.

Avant d'analyser quelques œuvres emblématiques de deux femmes futuristes, Rosa Rosà et Enif Robert, je voudrais préciser certains points.

Quand, pourquoi et pour quoi faire les femmes adhèrent-elles au futurisme? S'il est difficile de donner une réponse unique et valable pour toutes, on peut néanmoins donner des repères. Concernant la période d'adhésion, on constate que les femmes s'engagent tardivement : si l'on excepte le cas très particulier de Valentine de Saint-Point, qui adhère dès 1912-1913, les autres femmes arrivent pendant les années de guerre, lorsque les

seules artistes italiennes, ni les seules futuristes. Le catalogue a aussi été publié en français : Lea Vergine, L'Autre Moitié de l'avant-garde, 1910-1940, Paris, Des Femmes, 1982.

3. Silvia Contarini, La Femme futuriste. Mythes, modèles et représentation de la femme dans la théorie et la littérature futuriste (1909-1919), Nanterre, Presses Universitaires de Paris Ouest, 2006.

4. Le célèbre manifeste « Reconstruction futuriste de l'univers », signé Depero et Balla, publié le 11 mars 1915 est emblématique de la caractéristique essentielle du futurisme: l'ambition d'une transformation globale de l'individu et de son environnement, et l'annonce de l'avènement d'une humanité nouvelle dans un monde nouveau.

5. Roland Barthes, Roland Barthes, Paris, Seuil, 1975, p. 58. En fait, Barthes considère cette qualité comme une forme de chantage car les œuvres semblent dire : «Aimez-moi, gardez-moi, défendez-moi puisque je suis conforme à la théorie que vous défendez » (ibid.). 
hommes sont partis au front. Elles adhèrent surtout après la guerre lorsque le futurisme abandonne ses ambitions les plus politiques, visionnaires et globalisantes (l'ambition de reconstruire l'univers) pour se concentrer sur l'expression artistique. Autrement dit, la plupart des femmes participent au futurisme quand la réflexion sur la femme est close, réflexion qui était bien nourrie dans les années 1910 et qui atteint son paroxysme lors du débat sur le « problème » de la femme dans la revue L'Italia futurista (1916-1917).

Qu'est-ce qui motive les femmes? Les raisons de leur adhésion sont parfois de deux ordres : toutes expriment la volonté d'exalter les canons de la modernité et de rejeter les conventions du passé, mais certaines sont également convaincues que le futurisme encourage la remise en cause des modèles et des rôles sexuels. Elles pensent que l'homme nouveau futuriste ne peut avoir à son côté qu'une femme nouvelle et c'est cette femme qu'elles veulent contribuer à construire. Cette motivation est explicite chez Valentine de Saint-Point, Rosa Rosà et Enif Robert.

Quel est l'impact des femmes futuristes? Elles sont donc peu nombreuses au moment où la réflexion sur l'humanité nouvelle, femme et homme du futur, se développe au sein du futurisme et leurs propositions n'ont aucun espace. Même dans les années suivantes, leur rôle reste marginal dans l'élaboration théorique, esthétique et artistique du futurisme : pratiquement aucun manifeste artistique n'est signé par des femmes ${ }^{6}$; même des manifestes sur des sujets réputés typiquement « féminins » comme la mode, la cuisine ou la danse sont produits par des hommes. Aucune figure majeure de femme ne s'impose, à l'exception de Benedetta, dont les qualités artistiques sont indéniables, mais à qui le rôle d'épouse du chef confère à l'évidence un statut à part. En fait, les femmes n'ont pas vraiment eu d'influence au sein du futurisme; le contraire est moins vrai car le futurisme a pu avoir un impact sur leur créativité et sur leur pensée.

Revenons à l'adéquation des théories aux pratiques et à la pertinence de la forme artistique par rapport à la double spécificité, futuriste et féminine.

Les deux manifestes sur la femme publiés par Valentine de Saint-Point, prétendument en réponse au « mépris de la femme » marinettien (point 9 du "Manifeste de fondation du futurisme »), ont été vite oubliés ${ }^{7}$. Ils avancent

6. À l'exception de Benedetta (Cappa Marinetti), dont le nom apparaît dans trois manifestes collectifs tardifs : «Il manifesto dell'aeropittura » (1929), «La plastica murale futurista. Manifesto » (1934) et « Manifesto del gruppo futurista primordiali Sant-Elia » (1941). Pour la bio-bibliographie complète de Benedetta, voir la réédition de ses romans : Benedetta, $L e$ forze umane, Viaggio di Garara, Astra e il sottomarino (éd. et préface de Simona Cigliana), Rome, Edizioni dell'Altana, 1998.

7. Valentine de Saint-Point, Manifeste de la femme futuriste (1912) et Manifeste futuriste de la luxure (1913). Ces manifestes ont récemment connu plusieurs rééditions et ils sont également disponibles sur plusieurs sites internet. 
pourtant quelques idées intéressantes, comme l'affirmation que l'humanité n'est pas composée d'hommes et de femmes, mais d'individus avec des caractères à la fois féminins et masculins.

Cette même idée de la dissociation entre appartenance sexuelle et gender est avancée cinq années plus tard par les deux futuristes dont j'analyserai les œuvres, Rosa Rosà et Enif Robert. Il faut remarquer qu'elles ne citent pas Valentine de Saint-Point et qu'elles ne renvoient pas l'une à l'autre, alors qu'elles écrivent dans la même revue, à la même époque et sur le même thème de la femme nouvelle. Ces deux femmes futuristes, qui refusent explicitement les modèles féminins existants, ont d'autres points en commun (communs aussi à Valentine de Saint-Point) : la recherche, parfois confuse, d'une spécificité artistique féminine, et la quête d'une évolution personnelle, d'un dépassement de leur condition de femme ordinaire.

En particulier, Rosa Rosà élabore un concept de «femme de l'aprèsdemain », en abordant les questions centrales de la condition féminine et de l'identité sexuelle, en prônant à la fois l'émancipation et la libération des femmes et en se disant convaincue de l'imminence de changements politiques et sociaux et de profondes métamorphoses psychologiques, sexuelles et érotiques ${ }^{8}$. Quant à Enif Robert, elle met en évidence une contradiction fondamentale chez les hommes futuristes : en dépit des mots d'ordre du mouvement d'avant-garde, refus du passé, transformation du présent et tension vers l'avenir, les futuristes se révèlent conservateurs dès lors qu'il s'agit de changer la condition de la femme 9 . On retrouve ainsi dans leurs interventions théoriques des éléments qui distinguent totalement leur vision de la vision des hommes futuristes, c'est-à-dire, d'une part, l'intuition anti-essentialiste et le dépassement du binarisme, et d'autre part, le souci de se métamorphoser en un individu au-delà et au-dessus de la détermination sexuelle, de devenir un artiste, confusément configuré comme une sorte de troisième sexe, un être doté de sensibilité féminine et de talent masculin.

Un dernier élément réunit ces femmes, un élément qui cette fois les rapproche de leurs camarades hommes : le signe féminin reste associé à des principes négatifs - sentimentalisme, faiblesse, lâcheté, instinct, animalité - alors que le signe masculin reste associé aux qualités de courage, de

8. Rosa Rosà publie dans L'Italia futurista quatre articles : «Le donne del posdomani », vol. II, nº 18, 17 juin 1917; « Risposta a Jean-Jacques », vol. II, n 20, 1 juillet 1917; « Le donne cambiano, finalmente... », vol. II, nº 27, 26 août 1917; « Le donne del posdomani (II) », vol. II, n 30, 7 octobre 1917.

9. Enif Robert publie dans L'Italia futurista deux articles « Una parola serena », vol. II, $\mathrm{n}^{\circ}$ 30, 7 juillet 1917; "Come si seducono le donne. Lettera aperta a Marinetti », vol. II, $\mathrm{n}^{\circ} 36,31$ décembre 1917. Elle intervient ainsi dans un débat sur " le problème de la femme » qui a lieu dans la revue et au cours duquel certains hommes futuristes se sont exprimés de manière fort agressive, en qualifiant les femmes de stupides et frivoles, tout en considérant que la femme émancipée s'apparente à un homme manqué. 
force, d'indépendance et d'intelligence. Ce sont ces qualités que la femme nouvelle veut acquérir et ce sont ces qualités que prône le futurisme.

En conclusion d'un de ses articles polémiques relatifs à la définition de la femme, réagissant à l'ouvrage de Filippo Tommaso Marinetti Come si seducono le donne (Comment on séduit les femmes) ${ }^{10}$, Rosa Rosà affirmait que les femmes allaient acquérir « la conscience d'un libre "Moi" immortel qui ne se donne à personne et à rien » et s'insurgeait contre les hommes qui niaient encore que la femme pût avoir une âme ${ }^{11}$. Quelques mois plus tard, en 1918, elle publie le roman Una donna con tre anime (Une femme avec trois ames) ${ }^{12}$ : c'est une tentative explicite de « mise en œuvre » de sa vision de la femme nouvelle.

Ce roman s'inscrit dans le respect de certains paradigmes littéraires futuristes, telles que la synthèse et l'absence de psychologie (c'est un roman court où les personnages sont prototypiques) ou encore la simultanéité (deux actions se déroulent en parallèle : les réunions des scientifiques et les transformations d'une femme), tout en étant influencé par les intérêts pré-surréalistes (occultisme, ésotérisme) du groupe florentin (la pattuglia azzurra) auquel Rosà fait référence.

L'histoire est simple : grâce à une énergie exceptionnelle absorbée dans l'air (décharge électrique combinée à des substances chimiques), qui provoque une accélération temporelle, une femme au foyer, Giorgina Rossi, est projetée dans l'avenir et subit trois mutations successives. Sa première transformation l'amène à prendre conscience de sa sensualité et de son propre désir. La deuxième concerne l'esprit et libère la parole : la triste ménagère devient une oratrice exaltée qui veut transmettre le verbe de la nouvelle science. La dernière phase touche à la sphère de la création artistique et se manifeste dans l'écriture : la poésie est le stade ultime de la transformation du monde et partant, de la femme. En fait, ce n'est plus vraiment une femme, mais un être sans chair qui exprime dans un élan lyrique-conceptuel sa quête de libération de la matérialité, son désir d'envol loin du carcan terrestre, vers des dimensions autres.

10. Filippo Tommaso Marinetti, Come si seducono le donne, Firenze, Centomila copie, 1917. Il s'agit d'un «manuel» fort provocateur sur les manières de séduire les femmes, lesquelles ne pourront jamais résister aux techniques éprouvées de l'homme futuriste italien. 11. Rosa Rosà, «Le donne cambiano, finalmente... », op. cit. Quand l'édition française n'est pas indiquée, c'est toujours nous qui traduisons.

12. Rosa Rosà, Una donna con tre anime, Milano, Studio editoriale lombardo, 1918. Nouvelle édition Non c'è che te! Una donna con tre anime e altre novelle, Milano, Facchi, 1919. Ce roman a connu une seule réédition en italien (cf. note 2) et aucune traduction française. Nous en avons proposé une analyse plus approfondie dans « How to Become a Woman of the Future: Una donna con tre anime - Un ventre di donna ", dans Geert Buelens, Harald Hendrix et Monica Jensen (dir.), Futurisms. Precursors, Protagonists, Legacies, Lexington Books, 2012 (à paraître). 
Libération sexuelle, prise de parole, écriture poétique ne durent qu'un court laps de temps. Suffisamment long pour inquiéter les scientifiques et le mari qui veulent guérir la pauvre femme et la ramener à la normalité. Ils y parviennent. Dans le dernier chapitre, le professeur Ipsilon explique ce qui s'est passé comme une manifestation du rapport entre la " pré-conscience » et la réalité. Il affirme aussi que la femme du postdomani, l'après-demain, aura plusieurs âmes qui ne s'excluront pas l'une l'autre; la troisième âme ne pourra se manifester que dans un avenir très éloigné.

Ce roman a été lu et peut certainement se lire comme l'expression d'une double utopie, futuriste et féministe ${ }^{13}$. Il contient en effet des intuitions intéressantes. L'application des principes futuristes de simultanéité et de compénétration permet notamment de faire coexister des personnalités différentes et de contourner ainsi la contradiction entre plusieurs modèles de femme (sensuelle versus intellectuelle, féminine versus masculine). Il importe surtout de mettre en évidence l'intention d'imaginer une femme nouvelle qui soit l'application d'idées précédemment exposées et de préceptes futuristes.

Or, force est de constater que la métamorphose de la ménagère se clôt sur un échec. Pourquoi ne peut-elle pas réussir son évolution, même dans un texte de fiction? Rosà exprime là un doute sur les possibilités réelles de changement dans une temporalité à échelle humaine; ce n'est pourtant pas ce qu'elle affirme dans ses articles où elle se dit convaincue que grâce aux bouleversements apportés par la guerre, les femmes changent, même si cela ne plaît pas aux hommes ${ }^{14}$. Rosà exprime aussi un refus profond de caractéristiques réputées féminines auxquelles la femme de son époque reste associée. Ce doute et ce refus, que son volontarisme futuriste et sa conviction intellectuelle lui ont permis de surmonter dans ses textes théoriques, restent enracinés dans son imaginaire et conditionnent sa vision de l'avenir de la femme.

L'échec de sa transformation, explicite dans l'épilogue de l'histoire, est d'ailleurs décelable dans d'autres éléments narratifs et formels : structurellement, le récit oppose la science et le progrès (domaine masculin/pôle positif) à la norme et au passé (incarnés par la femme/pôle négatif); le genre choisi, le conte fantastique, permet d'imaginer une métamorphose qui n'a rien de vraisemblable et qui se déroule dans une dimension d'irréalité qui en atténue voire en annule la portée. Quant au personnage de la femme « future », celle que nous voyons prendre la parole devant la foule ressemble

13. Voir Lucia Re, « Scrittura della metamorfosi e metamorfosi della scrittura: Rosa Rosà e il futurismo », dans Emmanuelle Genevois (dir.), Les Femmes écrivains en Italie (18701920): ordre et libertés, Chroniques Italiennes, $\mathrm{n}^{\circ} 39-40$, Paris, Université Sorbonne Nouvelle, 1994, p. 311-327.

14. Rosa Rosà, « Le donne del postdomani (II) », op. cit. 
à une folle au discours délirant, et celle qui prend la plume pour écrire tel un grand poète inspiré produit de mauvaises pages au style maladroit. De plus, les motifs du vol, de la folie et du rêve, ressorts utilisés pour permettre à la femme de «s'élever » à la hauteur des hommes, bien qu'étant des topoi futuristes, peuvent être lus comme des moments d'évasion d'un quotidien médiocre que l'on ne saurait changer, des rêves de ménagère, l'expression de la frustration d'une femme qui n'a d'autre issue à sa morne vie que de fantasmer des aventures qu'elle ne vivra jamais.

Bref, en dépit de son intention et de son ambition, Rosa Rosà qui a pourtant élaboré le changement de la femme dans ses articles, n'exprime guère dans ce roman la conviction que le jour où la femme possédera trois âmes viendra vraiment. Ce manque d'assurance quant à la possibilité réelle pour une femme de s'épanouir dans le temps présent, ainsi que la difficulté de la quête d'une identité féminine aux contours encore trop imprécis, trouvent confirmation dans deux autres œuvres de Rosa Rosà datées de la même époque, une nouvelle et une planche motlibriste.

La nouvelle, intitulée Romantisme somnambule (puis L'Aquarium), compte une petite dizaine de pages ${ }^{15}$. Elle alterne deux « je» de narration. Le récit principal, qui a comme protagoniste un homme qui raconte son histoire, est précédé par quelques lignes d'incipit et suivi de quelques lignes d'épilogue, écrits par une femme. C'est elle qui explique que, la nuit précédente, elle s'est réveillée en sursaut; comme poussée par une force extérieure, elle s'est mise à écrire, presque sous la dictée, dans un style qui n'était pas le sien. Retournée se coucher, elle s'est réveillée le matin et a trouvé des feuilles couvertes de son écriture. Entre guillemets, se place alors le récit de l'homme. Il raconte ce qui s'apparente à un cauchemar : un ouragan épouvantable a précipité sa maison sous le niveau de la mer; la partie où il s'est retranché est restée étanche, le reste étant submergé, mais visible derrière des baies vitrées. C'est ainsi qu'il voit tous ses invités, figés par la mort dans les positions qu'ils avaient au moment du drame; sa femme est parmi eux, à côté d'elle se trouve un homme inconnu. Le narrateur ne supporte pas cette vision, il veut casser la vitre qui le sépare d'elle. Ici se termine son récit.

La narratrice reprend la parole pour conclure :

Ici se termine ce que j'avais écrit la nuit. Jusque-là, la chose n'aurait rien de bizarre, si ce n'est le fait que moi je suis une femme, alors que ces pages semblent être le récit de la terrible aventure d'un homme, dont l'âme a choisi

15. Rosa Rosà, "L'Acquario ", dans Non c'è che te!, op. cit. Une première version, réduite et intitulée « Romanticismo sonnambulo », avait paru dans L'Italia futurista, vol. II, $\mathrm{n}^{\circ}$ 17, 10 juin 1917. Nous citons à partir de la récente édition en anthologie : Cecilia Bello Minciacchi, Spirale di dolcezza + serpe di fascino. Scrittrici futuriste. Antologia, Naples, Bibliopolis, 2007, p. 181-189. 
cette voie pour se manifester dans des phrases précises, courtes et pesantes, avant de se perdre dans la folie ${ }^{16}$.

Il n'y a rien d'étrange, dit la narratrice qui croit sans doute comme Rosà à des phénomènes tels que la métempsychose, le somnambulisme et l'occultisme, à ce que l'âme d'un mort s'incarne dans le corps d'un être vivant en le forçant à s'exprimer à sa place. Elle trouve bien plus étrange qu'un homme ait choisi une femme pour se manifester et qu'une femme puisse assumer la parole et l'écriture d'un homme. Étrange, semble-t-elle suggérer, mais possible : une femme peut avoir l'âme d'un homme, son écriture. Constatons cependant qu'elle y parvient en rêve, pendant la nuit, dans un état second, au-delà de sa volonté et forcée par des énergies mystérieuses. Aussi, ce qu'elle/il raconte dans son cauchemar, ressemble beaucoup à une crise de jalousie... Il semble donc que dans cette nouvelle, tout comme dans le roman Une femme avec trois âmes, la femme exprime, d'une part, un désir très fort d'acquérir une âme masculine, qui est l'âme de la création et de l'écriture, et d'autre part, qu'elle n'ose affirmer son ambition que sous la forme d'un rêve dont elle souligne elle-même l'absurdité.

La planche motlibriste s'intitule Ricevimento - thé - signore - nessun uomo (Réception, thé, dames, aucun homme) ${ }^{17}$. C'est la représentation graphique et littéraire (formes géométriques simples et textes juxtaposés) d'un salon bourgeois où des dames se retrouvent conventionnellement pour le thé. En bas à gauche, devant la « porte» se trouve «moi » qui va sortir, en disant : « je crois que j'en ai assez ». Au centre, une « table basse » avec " petits fours et boissons »; en dessous, la mention " ennui, bâillements réprimés »; tout autour, des espaces d'où jaillissent des voix de femmes qui bavardent au sujet de la mode et de la villégiature, rapportent des potins ou se plaignent des hommes.

Le but de cette planche est principalement la contestation des valeurs bourgeoises ${ }^{18}$. Il convient de remarquer toutefois qu'elle prend pour cible des femmes, rien que des femmes, comme si elles seules véhiculaient ces valeurs. Rosa Rosà se range ainsi doublement du côté des futuristes : par le moyen artistique choisi (la planche motlibriste) et par une représentation de femmes superficielles et stupides. Ce qu'elle semble le plus condamner, c'est l'ennui qui se dégage des conversations entre femmes. Il y a un détail qui frappe : le titre prend soin de préciser « aucun homme». Comme si la

16. Ibid., p. 188.

17. Rosa Rosà, « Ricevimento - thé - signore - nessun uomo », L'Italia futurista, vol. II, $\mathrm{n}^{\circ} 35,9$ décembre 1917. On peut en voir une reproduction sur http://www.futur-ism.it/ esposizioni/ESP2009/ESP20091109_NY.htm

18. C'est l'interprétation qu'en donnent Cecilia Bello Minciacchi, op. cit., p. 160-161, et Giancarlo Carpi, op. cit. L'un et l'autre soulignent des éléments formels (le salon est vu du haut; il n'y a pas de jeux typographiques; les personnes et les tables sont dessinées en triangles et cercles; les mots forment des lignes ondulées), mais ils ne s'arrêtent pas sur le clivage homme-femme pourtant énoncé dans le titre. 
présence masculine était indispensable pour élever le niveau de la conversation parce que sans les hommes les femmes en seraient réduites à se raconter banalités et sottises.

Pas plus que les précédentes, cette œuvre n'exprime de confiance dans les moyens qu'ont les femmes de parvenir à modifier leur condition et de s'émanciper intellectuellement. Elle exprime même quelque chose de plus profond, l'impossibilité pour les femmes de parvenir à changer leur situation collectivement et en se soutenant mutuellement. Leur salut - individuel - viendra des hommes. Ce n'est pourtant pas ce que Rosa Rosà écrivait dans ses articles.

Je m'arrêterai moins longuement sur Un ventre di donna: romanzo chirurgico (Un ventre de femme : roman chirurgical), roman signé Marinetti et Enif Robert ${ }^{19}$, mais dont Robert est la véritable auteure, seules des lettres de Marinetti étant intercalées dans son texte. Il est vrai que la signature de Marinetti en couverture est valorisante...

Ce roman raconte lui aussi l'histoire de la métamorphose d'une femme ordinaire en femme futuriste. Dans sa préface, intitulée Coraggio + Verità, Enif Robert place explicitement ce livre dans le cadre du débat sur la femme qui a eu lieu dans les colonnes de L'Italia futurista ${ }^{20}$. Il en est donc un prolongement littéraire. En passant en revue tout ce que la femme ne doit pas être (ni rebelle, ni coquette, ni sentimentale, bien sûr), Robert affirme que la femme futuriste ne sera pas détachée de la réalité, ni enfermée dans une dimension esthétique, spirituelle ou onirique; elle mentionne comme des exemples à ne pas suivre les scrittrici azzurre ${ }^{21}$ (dont Rosà fait partie) et leurs « rêves ». La femme futuriste doit avoir le courage de raconter la vraie vie, la vérité, aussi laide soit-elle. C'est ce que Robert fait, ou fait croire qu'elle veut faire.

Un ventre de femme, roman-vérité donc, se présente au premier abord comme le journal intime d'Enif Robert, chronique d'une très longue maladie que l'auteure a réellement vécue. Mais au travers de celle-ci s'ébauche progressivement une métamorphose plus métaphorique de la

19. Filippo Tommaso Marinetti, Enif Robert, Un ventre di donna: romanzo chirurgico, Milan, Facchi, 1919. La préface porte la date de mars 1918. Les événements racontés sont situés en 1915-1916. Rappelons que le débat se déroule entre 1916 et 1917, que la publication de Comment on séduit les femmes de Marinetti intervient en 1917, suivie de la publication du roman de Rosà, Une femme avec trois âmes (1918). Un ventre de femme peut être considéré comme le volet de clôture.

20. La préface commence ainsi : « Nous n'avons pas encore bien compris, il me semble, ce que veut dire "FEMME FUTURISTE" ", ibid., p. XI.

21. On appelle Pattuglia azzurra ("Patrouille bleue») un groupe de futuristes florentins que l'on considère comme des précurseurs du surréalisme, en particulier pour leur exploration du monde onirique. 
protagoniste du roman, laquelle affirme, presqu'au début de l'histoire, qu'elle se sent « peu femme » parce qu'elle est davantage attirée par l'art que par l'amour : «J'aurais été un peu peintre, un peu poète si j'étais née homme. L'amour ne me suffit pas. Je me sens vraiment, en ce moment, peu femme ${ }^{22}$. » L'amour est aux femmes ce que l'art est aux hommes : une qualité naturelle. Si une femme veut devenir artiste, elle doit transiter du féminin au masculin.

La maladie affecte donc le corps de la protagoniste dans sa partie la plus biologiquement féminine : les organes de la procréation. La science officielle et passéiste ne parvient pas à la guérir parce qu'elle est incapable de comprendre que la femme nouvelle n'est pas réductible à sa nature utérine, tota mulier in utero. Un corps, même très féminin, peut être accompagné d'un esprit masculin. C'est le constat désemparé que dresse le médecin : la malade a un "cerveau » trop masculin dans un corps trop féminin. Ces propos sont ressentis comme un compliment par la malade, fière d'être parvenue à faire coexister en elle une cérébralité d'homme et la sensualité d'une femme. Cet assemblage de prérogatives antinomiques a toutefois son prix à payer : sa vie est en danger deux ans durant et la gravité de l'infection exige l'ablation de ses organes génitaux féminins.

La doctrine futuriste comprend-elle les problèmes de la femme nouvelle? Les livres futuristes remplacent la Bible sur la table de chevet de la malade, Marinetti, depuis le front, l'encourage à la bataille, en détaillant ses conseils d'abord dans une " cure futuriste » à base d'optimisme et de volonté, puis dans un " Manuel thérapeutique du désir-imagination». La malade décide de canaliser son effort dans celle qu'elle considère comme la meilleure des thérapies, l'écriture futuriste. La création littéraire représente une étape essentielle de sa transformation/guérison, elle marque la transition vers une dimension à ses yeux supérieure, celle de l'artiste :

\footnotetext{
Mon but? Le voici : le plus absurde, le plus difficile, celui de devenir... une écrivaine futuriste! Je vais écrire, tout de suite [...] un volume de mots en liberté, intitulé "sensations chirurgicales" et je l'enverrai à Marinetti. Allez, au travail ${ }^{23}$.
}

Elle écrit quelques pages de ces "Sensations chirurgicales », puis d'autres mots en liberté ( Conversation entre le soleil et mon ventre »). L'insertion de ces créations - pas très intéressantes sur le plan littéraire dans un récit jusque-là autobiographique ou épistolaire souligne qu'il s'agit bien de l'histoire de la transformation d'une femme conventionnelle en femme artiste et futuriste, et d'une œuvre de fiction. Le roman se conclut d'ailleurs par un changement de registre déconcertant. Le récit à la première personne de la malade Enif Robert s'arrête brusquement. 
La protagoniste du dernier chapitre est une certaine princesse de Ruderis, atteinte d'une maladie au ventre que la science ne sait pas guérir mais dont les causes principales sont les nombreux accouchements et le port de corsets trop serrés. Les scientifiques sont désemparés. Le vieux prince interdit que l'on touche au corps de sa jeune épouse. Coup de théâtre : un brave Ardito futuriste le poignarde. La jeune femme enfin libérée du vieux mari se libère aussi de sa maladie toute féminine en se faisant opérer. La dernière scène la montre au balcon, nue, belle, résolue, admirée par de valeureux futuristes.

Ce roman représente l'évolution de la femme comme un processus long et douloureux; il représente la féminité comme une maladie; il suggère enfin que la nouvelle identité de la femme se forge en expérimentant la difficile coexistence de prérogatives masculines et féminines, la cohésion entre corps, âme et esprit. Enif Robert imagine plus précisément l'association d'un corps qui préserve une sensualité féminine, sans pour autant être destiné à la procréation, avec un esprit virilisé permettant à la femme de se réaliser dans la création. Remarquons pourtant que l'adhésion aux préceptes futuristes, l'accès à la création artistique et l'ablation génitale, les trois expériences qui ont rendu la protagoniste du roman moins femme en la rapprochant de l'homme, et qui l'ont ainsi en quelque sorte préparée à se métamorphoser en femme futuriste, n'ont pas été suffisantes à l'accomplissement de son évolution.

D'ailleurs, en dépit de l'appel initial à la réalité et à la vérité, le singulier épilogue indique que la maladie de la femme tout autant que sa guérison doivent se lire de manière allégorique. La protagoniste Enif Robert n'accomplit pas jusqu'au but le processus de guérison-transformationlibération que réussit en revanche à accomplir une femme chimérique. La femme futuriste qui apparaît dans le final n'est pas celle dont nous avons suivi les complexes étapes de transition, c'est un personnage peu vraisemblable qui, comme dans un conte fantastique, se libère d'un coup en échangeant un vieux mari contre de jeunes soldats, les contraintes des corsets contre la liberté de la nudité. Autrement dit, la transformation en femme futuriste n'aboutit que dans une dimension fictionnelle, métaphorique et explicitement irréelle, alors que dans la réalité ou dans ce qui est supposé être réel, cette transformation, laborieuse et périlleuse, n'aboutit pas. Remarquons aussi que la femme du futur qui en résulte, une jeune princesse nue, belle et admirée par des héros, est fort éloignée du modèle de femme idéalisé par Enif Robert, qui cherchait plutôt à configurer une artiste aux caractères hybrides, masculins et féminins.

D'un point de vue formel, ce « roman chirurgical » se caractérise par une succession de genres et de registres. Barbara Zecchi a très bien analysé l'évolution du genre littéraire : le texte initial de Robert en forme de journal intime (genre féminin) cède le pas à l'échange épistolaire avec Marinetti (genre mixte) pour parvenir à la fiction (genre réputé masculin). L'évolution 
du genre littéraire accompagne donc l'évolution du gender, la transition du féminin au masculin ${ }^{24}$.

Il convient enfin de remarquer que tout au long du roman le parallèle s'établit entre la bataille de Robert et la bataille de Marinetti ( « Finalement, écrit Marinetti, je suis dans votre situation. Vous êtes clouée au lit, moi dans une tranchée boueuse $\left.{ }^{25} \gg\right)$. La maladie de l'une est assimilée à la guerre de l'autre, les batailles intimes aux batailles au front. Mais la femme mène sa lutte dans l'espace clos de la chambre sans sortir de la sphère privée, tandis que l'homme mène sa bataille sur un terrain de confrontations politique, sociale et publique; la femme subit passivement et individuellement, l'homme est dans l'action collective et exaltante. Cette différence, qui est une différence dans les valeurs associées au genre et qui repose sur une conception essentialiste, se révèle d'emblée; aux premiers signes de son infection, la narratrice s'exclame : "Quel dégoût que d'être (nota bene : «être » pas « avoir ») un utérus souffrant, alors que tous les hommes se battent ${ }^{26}$ ! ». Les guerres qui comptent sont celles qui « hygiénisent» le monde en le nettoyant des faiblesses du féminin, les guerres où s'exaltent les valeurs viriles.

Bref, ce sont le reniement profondément ancré du genre féminin et l'adhésion inconditionnelle au genre masculin qui expliquent les raisons de l'inaboutissement de la métamorphose de la protagoniste : elle voulait préserver ses qualités féminines, tout en les méprisant; elle voulait devenir femme artiste, mais en se muant en homme. Elle se disait persuadée, tout comme Robert l'affirme dans ses articles, que l'assemblage de prérogatives des deux genres est une voie praticable, mais le résultat montre le contraire : le processus entamé s'interrompt. Finalement, dans sa vision romanesque, Robert ne se départit pas des deux stéréotypes féminins les plus éculés et binaires : d'une part, la femme utérine conventionnelle, objet du mépris futuriste (et du sien); de l'autre, la femme jeune, belle et libre, objet de la convoitise des futuristes.

Rosa Rosà et Enif Robert dans leurs œuvres de fiction ont voulu penser et en même temps imaginer la métamorphose de la femme. Elles sont conscientes du double enjeu futuriste et féminin sur les plans thématique et

24. Barbara Zecchi, «Il corpo femminile trampolino tra scrittura e volo. Enif Robert e Biancamaria Frabotta: settant'anni verso il tempo delle donne », Italica, $\mathrm{n}^{\circ}$ 4, 1992, p. 505-518.

25. Filippo Tommaso Marinetti et Enif Robert, Un ventre di donna: romanzo chirurgico, op. cit., p. 171. Les métaphores guerrières sont nombreuses et fondamentales; j'ai abordé cet aspect dans "Guerre maschili/guerre femminili: corpi e corpus futuristi in azione/ trasformazione ", dans Federico Luisetti et Luca Somigli (dir.), A Century of Futurism: 1909-2009, Annali di italianistica, n ${ }^{\circ} 27$, Chapel Hill, The University of North Carolina, 2009, p. 125-138.

26. Filippo Tommaso Marinetti et Enif Robert, Un ventre di donna: romanzo chirurgico, op. cit., p. 25. 
formel. Si elles ne sont pas les seules femmes futuristes qui produisent des œuvres, en ce moment de débat théorique et idéologique sur les rôles sexuels, elles sont les seules à tenter de concilier réflexion théorique et création en les imbriquant. Leurs tentatives restent remarquables à plusieurs égards, même si elles demeurent contradictoires et limitées.

Ce qui interpelle, c'est qu'en dépit de leur intention, et contrairement à ce qu'elles ont exprimé dans leurs textes théoriques, elles n'ont pas réussi à envisager de véritable modèle positif de femme, une femme du futur crédible. La raison semble se situer dans la remise en cause partielle et partiale des prérogatives de genre. En fait, Rosà et Robert ont démoli le concept de féminin et ses attributions conventionnelles, mais elles n'ont pas dissocié « femme » et « féminin »; en même temps, elles n'ont pas démantelé le concept de masculin en laissant l'homme sur son piédestal. C'est la femme qui est un « problème » (c'est le titre de la rubrique qui accueille le débat sur la femme dans L'Italia futurista); l'homme reste la perfection, le modèle idéal à atteindre : intelligence, esprit, créativité, courage, force, tout lui appartient; la femme ne peut que s'efforcer de conquérir ses qualités, de l'imiter, de l'égaler. C'est une vision conventionnelle des genres, qui plus est tout à fait conforme à l'idéologie futuriste; elles n'ont pas su s'en démarquer.

L'aura « virile » de Marinetti, le chef de file incontesté, joue sans doute un rôle non négligeable. Comment oublier que Marinetti est le «maître » quand on regarde Portrait de Marinetti, les mots en liberté que lui dédie sa domestique, Marietta Angelini (qui signe d'ailleurs avec une certaine fierté « 1a cameriera di Marinetti ») ${ }^{27}$ ? Un « 1 » phallique, qui devait être imprimé en rouge, trône au centre de la planche. Angelini est aussi l'auteure de la planche intitulée Portrait de Cangiullo, qui a une structure similaire : une figure phallique centrale formée de mots exalte la beauté et la sensualité virile du futuriste Cangiullo, grâce auquel ces compositions, au demeurant très simples dans la conception comme dans la réalisation, sont publiées. Ce même Cangiullo, qui les définit comme des « compositions géniales 》, les accompagne d'une lettre adressée aux écrivaines les plus célèbres de l'époque (Matilde Serao, Grazia Deledda, Ada Negri et Teresah), les invitant à suivre l'exemple d'Angelini. C'est-à-dire l'exemple d'une femme dont les œuvres sont un hymne à la virilité et à la puissance des maîtres, doublé d'un hommage aux hommes qui l'ont hissée de sa condition de subordonnée au rang de poète. Ces mots en liberté sont aujourd'hui encore considérés comme l'expression d'un singulier talent artistique futuriste féminin ${ }^{28}$. Ce n'est pas mon avis.

27. Marietta Angelini, Ritratto di Marinetti et Ritratto di Cangiullo, Vela latina, vol. IV, $\mathrm{n}^{\circ}$ 5, 12 février 1916. Les planches peuvent être visualisées sur le site http://www.paviatricolore.org/artelettere.htm.

28. Cecilia Bello Minciacchi écrit : «De plein droit, Marietta, qui était analphabète - tout au plus autodidacte, mais absolument illettrée -, fut la première femme poète motlibriste 
Ils sont au contraire emblématiques d'un problème qui concerne toutes les femmes futuristes : l'admiration indéfectible pour l'homme et ce qu'il représente, une admiration explicite chez Enif Robert, plus intériorisée et refoulée chez Rosa Rosà. À cet homme dépositaire par nature de tout ce qui possède une valeur se confronte une femme toujours représentée en négatif, une femme que l'homme méprise et que la femme, malgré ses meilleures intentions, ne peut apprécier. Ces écrivaines futuristes ne veulent pas appartenir à une catégorie dévalorisée. Elles s'en distinguent alors, se démarquent du collectif féminin en privilégiant une démarche individuelle, et tentent d'imaginer leur propre transformation en transcendant l'appartenance de genre. Mais qu'il est difficile, quand on n'aime pas les femmes, de s'imaginer en femme nouvelle, d'écrire en femme nouvelle!

On comprend pourquoi la rupture avec le passé et la création d'un «type supérieur de femme » ne restent qu'une aspiration, plus facile à théoriser, en faisant fi des apories, qu'à concrétiser dans des représentations de modèles positifs et nouveaux, d'hommes et de femmes.

Silvia Contarini Université Paris Ouest Nanterre La Défense, CRIX (EA 369)

[...]. La caractéristique de ces deux planches motlibristes est une spontanéité presque absolue unie à une admiration pleine d'affection [n.d.a. pour Marinetti et Cangiullo]» (op. cit., p. 81-82). Elle ajoute : «Ces deux planches avaient un double privilège : celui de représenter une nouveauté absolue dans le domaine de la production poétique féminine et celui de proposer dans une synthèse verbo-graphique les traits intellectuels, caractériels et iconographiques du "type futuriste" » (Ibid., p. 83). 